\title{
Publicar sobre Educación Ambiental y Educación para la Sostenibilidad, ¿dónde?
}

\author{
Antonio Navarrete \\ Universidad de Cádiz. Facultad de Ciencias de la Educación. Cádiz España. \\ antonio.navarrete@uca.es \\ ORCID:https://orcid.org/0000-0001-7487-5617 \\ Pilar Azcárate \\ Universidad de Cádiz. Facultad de Ciencias de la Educación. Cádiz España. \\ pilar.azcarate@uca.es \\ ORCID:https://orcid.org/0000-0002-1306-5140 \\ Rocío Jiménez-Fontana \\ Universidad de Cádiz. Facultad de Ciencias de la Educación. Cádiz España. \\ rocio.fontana@uca.es \\ ORCID:https://orcid.org/0000-0003-2111-1132 \\ José Ma Cardeñoso \\ Universidad de Cádiz. Facultad de Ciencias de la Educación. Cádiz España. \\ josemaria.cardenoso@uca.es \\ ORCID:https://orcid.org/0000-0003-3905-9117 \\ Esther García-González \\ Universidad de Cádiz. Facultad de Ciencias de la Educación. Cádiz España. \\ esther.garcia@uca.es \\ ORCID:https://orcid.org/0000-0003-0995-9798
}

[Recibido: 12 Marzo 2019. Revisado: 21 Marzo 2019. Aceptado: 21 Marzo 2019]

Resumen: Presentamos un estudio cuyo objetivo es justificar la necesidad de creación de nuevos espacios que permitan la comunicación de investigaciones y experiencias en torno a la Educación Ambiental y la Educación para la Sostenibilidad. Estos espacios son una vía para hacer llegar tanto a la comunidad científica como a la sociedad, información sobre la situación de crisis socioambiental pero también sobre posibles soluciones a la misma.

Para aproximarnos al estado de la cuestión, se han revisado un total de 1743 artículos para el periodo 2014-2018, en ocho revistas del ámbito educativo y una del campo de las ciencias experimentales. Asimismo, se analizó la política y el alcance de estas revistas, y sus secciones para caracterizar mejor el tipo de publicaciones que recogen. Los resultados muestran que la presencia de estos temas es relativamente baja en las revistas analizadas. De manera que es necesario ampliar los espacios y posibilidades de publicación. Para ello es importante que las publicaciones existentes aumenten su receptividad a este tipo de trabajos, pero también es imprescindible la creación de nuevas publicaciones como la que presentamos en este trabajo: Revista de Educación Ambiental y Sostenibilidad.

Palabras clave: Educación Ambiental; Educación para la Sostenibilidad; Publicaciones Científicas. 


\section{Post about Environmental Education and Education for Sustainability, where?}

Abstract: We are presenting a study with the objective of justifying the need to create new publications to promote communications of different research and experiences involving Environmental and Sustainable Education.

These publications are a means to communicate to the scientific community as well as to society in general information about the social-environmental crisis but also to offer possible solutions to this situation. In order to collect data on the subject a total of 1743 articles publish between 2014-2018 have been revised, in eight different journals from this area of education as well as one from the area of the experimental sciences. We analyzed the politics and the scopes of these journals and their different sections to characterize better the type of publications involved. The results have shown that the presence of these themes is relatively low in the journals we have gone through. There is a need to broaden spaces and possibilities to publish. To do so it is important that existing journals increase their responsiveness to this kind of work, but also absolutely necessary to create new journals like the one we propose in this article: Revista de Educación Ambiental y Sostenibilidad.

Keywords: Environmental Education; Education for Sustainability; Scientific Publications.

Para citar este artículo: Navarrete, A., Azcárate, P., Jiménez-Fontana, R., Cardeñoso, J. M. y GarcíaGonzález, E. (2019) Publicar sobre Educación Ambiental y Educación para la Sostenibilidad, ¿dónde?. Revista de Educación Ambiental y Sostenibilidad 1(1), 1303. doi: 10.25267/Rev_educ_ambient_sostenibilidad.2019.v1.i1.1303

\section{Presentación del estudio}

El objetivo del presente artículo es justificar la necesidad de creación de nuevos espacios que permitan la comunicación de investigaciones y experiencias en torno a la Educación Ambiental y Educación para la Sostenibilidad. Para aproximarnos al estado de la cuestión se han revisado un total de 1743 artículos para el periodo comprendido entre 2014-2018, publicadas en ocho revistas del ámbito educativo y una de ellas del campo de las ciencias experimentales. Para poder dar una imagen más completa de las revistas consideradas, se ha analizado la política y alcance de estas revistas, así como las secciones, con el objetivo de caracterizar mejor el tipo de publicaciones recogidas.

El criterio de selección de los artículos ha sido que apareciera en el título, resumen o palabras clave el término Educación Ambiental y/o Educación para la Sostenibilidad. (en adelante EA/ES). Para el caso de la selección de las revistas, se han seleccionado según (el impacto) y procurando la representatividad de diversas áreas de conocimiento que son identificadas por la comunidad científica como más afines con la temática. Este estudio tiene como referente previo el realizado por JiménezFontana y García-González (2017) en el cual se analizó la visibilidad de la Educación ambiental y la Educación para la Sostenibilidad en las publicaciones al uso desarrolladas en España sobre Didáctica de las Ciencias en el período 2006-2015. Los resultados mostraron la baja presencia de este tipo de estudios en el cómputo global de las publicaciones analizadas.

La Educación Ambiental viene demostrando hasta el momento ser capaz de generar propuestas adecuadas a un mundo en rápida evolución, de tener reflejos y ser flexible ante los nuevos retos y contextos cambiantes (Mayer, 1998). En este sentido, no podemos asumir que exista una única concepción de Educación Ambiental, sino todo lo contrario. Según García (2002), es un ámbito de pensamiento y acción en el que predomina el debate y la heterogeneidad; con paradigmas teóricos, estrategias de 
actuación, practicantes y escenarios muy diversos. Se trata de un ámbito pluriparadigmático, con diversas tendencias, todas ellas vigentes. Estamos pues ante un panorama muy heterogéneo. Tal disparidad puede ser enriquecedora, pero dificulta enormemente la reflexión en la acción y la evaluación de las actuaciones. Queda de manifiesto en que, si bien se han desarrollado muchas prácticas en el ámbito de la EA, de distinta naturaleza, existe una descompensación importante respecto al bajo grado de aporte reflexivo, de construcciones teóricas $y$ fundamentaciones metodológicas asociadas a dichas prácticas (Benayas, Gutiérrez y Hernández, 2003).

Una posible forma de argumentarlo, pero no tanto de justificarlo, podría ser que la investigación en EA, al tener como objeto de estudio a la propia EA, se constituye por las relaciones entre los aspectos ambientales y educativos. En este sentido, para algunos investigadores sus fronteras no son tan claras, por los distintos sesgos con los que ha sido abordada, como el ecológico o el antropocéntrico. Esto ha originado confusiones como, por ejemplo, el hecho de centrar la atención en prácticas conservacionistas que, si bien corresponden a una perspectiva en la EA, no constituyen por sí mismas este tipo de educación (Flores, 2012). Desde este posicionamiento reduccionista, la EA ha estado sobre todo vinculada con la adquisición de conocimientos sobre el ambiente, dando muy poco espacio al desarrollo de competencias éticas, críticas y estratégicas, siendo necesario reconocer los obstáculos existentes en el contexto social y educativo en el que la EA/ES ha intentado practicarse, así como la falta de recursos para su desarrollo (Sauvé, 1999).

Bien es cierto que, con el desarrollo progresivo que ha venido experimentando la investigación en EA/ES desde el mundo universitario, se ofrece a la sociedad argumentaciones más sólidas y respuestas más fundamentadas acerca de su utilidad. Argumentaciones menos basadas en especulaciones e intuiciones y más asentadas en la puesta en valor de argumentos empíricos para justificar y documentar las afirmaciones, probar los logros, comparar la evolución y justificar los cambios a diferentes niveles (Benayas, Gutiérrez y Hernández, 2003). Es decir, el rol que ha empezado a jugar en los últimos años la investigación sistemática y continuada en todo este ámbito constituye un factor digno de consideración que ha de revertir sobre el propio campo con efectos positivos. No obstante, a pesar del fuerte carácter interdisciplinar de la EA/ES, no hay apenas equipos de investigación integrados por personas de diferente procedencia disciplinar. Así, los diferentes autores se centran aisladamente en fuentes documentales con mayor proximidad al área de conocimiento de origen. A estas alturas todavía es difícil encontrar a nivel nacional citas de revistas propias del campo de la Didáctica de las Ciencias que publiquen artículos sobre EA/ES de autores que proceden del campo de la pedagogía. De igual modo ocurre con autores que proceden de las Ciencias Ambientales, en los que no se localizan apenas citas de revistas de corte más didáctico o pedagógico. Tampoco abundan las citas cruzadas de ambos sectores. Además, cuando se realizan revisiones globales, se suele ignorar la producción de otros sectores (García, 2002).

Sin embargo, cabría esperar que ocurriese, pues los científicos no sólo producen conocimiento en su ámbito de especialización, además son usuarios del conocimiento que otros han producido en campos distintos. Así, los procesos de transferencia que pueden generar innovaciones se producen en redes de conocimiento con diferentes nodos de innovación (Echeverría, 2008). Por tanto, son indudables los aportes y 
contribuciones que todavía ha de acarrear la investigación continuada y sistemática para el desarrollo, crecimiento y consolidación de la EA/ES en nuestro país (Benayas, Gutiérrez y Hernández, 2003). Este debate tiene mucho que ver no solo con el escaso peso del mundo académico en un campo controlado por instituciones sociales que, o no se coordinan entre sí, o tienen intereses contrapuestos; sino también debemos añadir la cuestión del perfil profesional del educador ambiental (García, 2004). Ambas cuestiones limitan la perspectiva crítica y la idea de realizar una EA/ES integradora, aspectos determinantes para afrontar realidades complejas y sistémicas (Colom y Sureda, 1989).

Pero hay otro aspecto que consideramos de gran calado y que pretendemos poner de manifiesto con este trabajo, relacionado con las posibilidades de publicación de artículos de esta temática. En esta línea, en un estudio previo (Novo y Bautista-Cerro, 2012), las autoras concluyen que se detecta una falta de difusión de experiencias de buenas prácticas y de investigaciones empíricas y aplicadas. Según argumentan, pudiera parecer que se deba a una escasez de realizaciones en este campo, al tiempo que reconocen que es una cosa poco probable. Así, tal vez responda a las dificultades propias de los requerimientos de las revistas científicas, que a veces llevan a muchos expertos a derivar sus artículos hacia publicaciones menos especializadas (sobre todo a quienes trabajan en los primeros niveles de la educación formal y en la no formal, sectores donde existen numerosas y riquísimas experiencias en este campo). En un estudio posterior al mencionado (Jiménez-Fontana y García-González, 2017) se concluye que la proporción de publicaciones sobre temas de EA/ES en el área de Didáctica de las Ciencias Experimentales, donde se desarrollan muchas trabajos de este campo, es escasa.

Desde nuestro entender, la puesta a disposición en acceso abierto de los resultados de investigación y de las publicaciones científicas incrementa el uso e impacto de los contenidos y mejora la calidad de la investigación. Además, facilita una transferencia de conocimiento a la sociedad (Ferreras-Fernández y Merlo-Vega, 2015) democratizando el saber. Es constatable como tiene lugar una mejora notable del funcionamiento de la comunicación científica y también genera beneficios directos sobre la sociedad. Ambos planos, además, se retroalimentan.

Por este motivo, creemos que es adecuado realizar un nuevo estudio que analiza la presencia y representación de publicaciones de este ámbito en revistas del entorno educativo y del campo de las ciencias experimentales.

\section{Metodología}

La investigación desarrollada trata de analizar la presencia y características de artículos de Educación Ambiental y Educación para la Sostenibilidad en publicaciones científicas del ámbito educativo tanto generales como específicas, durante los últimos cinco años, es decir, el periodo de 2014-2018.

El estudio realizado tiene una naturaleza exploratoria-descriptiva, adecuada para el análisis documental del contenido de las revistas consideradas. Los estudios descriptivos recogen y analizan información con fines exploratorios y proporcionan hechos y datos que permiten obtener información sobre el problema formulado (Newby, 2010). 
En nuestro caso para obtener la información de las revistas analizadas hemos optado por una estrategia de análisis de contenido desde el punto de vista semántico. Consideramos importante emplear esta estrategia porque nos da la posibilidad de aproximarnos a las ideas expresadas en los diferentes documentos, centrándonos especialmente en aspectos descriptivos (López Noguer, 2002).

El análisis de contenido, es aplicable a todas las formas de comunicación y sectores de las ciencias humanas evaluando críticamente los hallazgos existentes en directo (Krippendorff, 2013, Ortega y Galhardi, 2013).

Tomando como referencia estudios previos realizados en este ámbito (Novo y Bautista-Cerro, 2012; Jiménez-Fontana y García-González, 2017) se han diseñado las siguientes cuestiones de investigación:

1. Conocer la presencia de las temáticas de EA/ES en publicaciones del ámbito educativo para el periodo comprendido entre 2014-2018.

2. Analizar la representatividad de las temáticas de EA/ES dentro de dichas publicaciones así como su política y alcance.

3. Caracterizar el tipo de trabajos publicados en el ámbito de la EA/ES.

La muestra considerada incluye una representación de revistas del ámbito educativo, general y específico, pues se considera que son un campo en el que también pueden tener cabida las temáticas señaladas. En la selección de las revistas se tuvieron en cuenta, asimismo, la significatividad de la misma dentro del ámbito educativo, su trayectoria en relación a las temáticas de interés para el estudio y por último que estuvieran representadas todas las áreas de conocimiento pertenecientes al ámbito educativo.

Así pues, las revistas seleccionadas fueron nueve. Cuatro de ellas más globales, Revista de Psicodidáctica, Revista de Educación, Revista Iberoamericana de Educación, Revista deInvestigación en la Escuela; las otras cuatro en relación con ámbitos más específicos, Enseñanza de las Ciencias, Revista de Didáctica de las Ciencias Experimentales y Sociales, Revista Eureka sobre Enseñanza y Divulgación de las Ciencias y Bolema: Boletim de Educação Matemática. También se ha analizado una revista relacionada con el ámbito de las ciencias experimentales, Ecología Austral.

Para la selección de los artículos se realizó una revisión exhaustiva de los diferentes volúmenes de las revistas citadas en el periodo comprendido entre 2014-2018, atendiendo a título, resumen y palabras clave. Se consideraron exclusivamente los textos donde aparecía de forma explícita en alguna de estas tres partes: Educación Ambiental, Educación para la Sostenibilidad, Sostenibilidad curricular o Ambientalización curricular.

Una vez identificados los artículos se organizaron en los diferentes años de publicación y se clasificaron en torno a su orientación: investigación, innovación o fundamentación. La primera hace referencia a investigaciones desarrolladas en la práctica de diseños y uso de recursos en los distintos ámbitos y contextos de la EA/ES, la relativa a innovación tiene que ver con propuestas de intervención innovadoras en este ámbito y, por último, la de fundamentación hace referencia a estudios teóricos y epistemológicos relacionados con la EA/ES. Para esta clasificación usamos el análisis del contenido de los resúmenes de las diferentes aportaciones. 
Se analizaron un total de 1743 artículos. El procesado informático de los mismos se realizó con el software de análisis cualitativo QRS N-Vivo9 para agrupar la información procedente de distintas fuentes, en este caso las diferentes revistas, en ítems diferenciados y además poder concretar frecuencias de presencia.

\section{Resultados y discusión}

La investigación desarrollada nos ha permitido obtener información de distinto signo, siempre relevante, relativa a las diferentes cuestiones de investigación, en torno a las cuales se presentan los resultados obtenidos.

Cuestión 1. Conocer la presencia de las temáticas de EA/ES en publicaciones del ámbito educativo y de las ciencias experimentales para el periodo comprendido entre 20142018.

Tal como puede observarse en la figura 1, los artículos cuya temática central es la EA/ES, suponen solo un 3\% de los trabajos publicados frente al $97 \%$ del resto de temáticas, lo cual deja de manifiesto la escasa presencia de los mismos en las revistas analizadas.

Este dato resulta cuanto menos alarmante, pues da a entender que la situación de emergencia planetaria, que responde a una crisis socioambiental de calado profundo, no ha permeado lo suficiente en el ámbito educativo, que es quien a través de sus publicaciones difunde las investigaciones e intervenciones relacionadas con estas cuestiones.

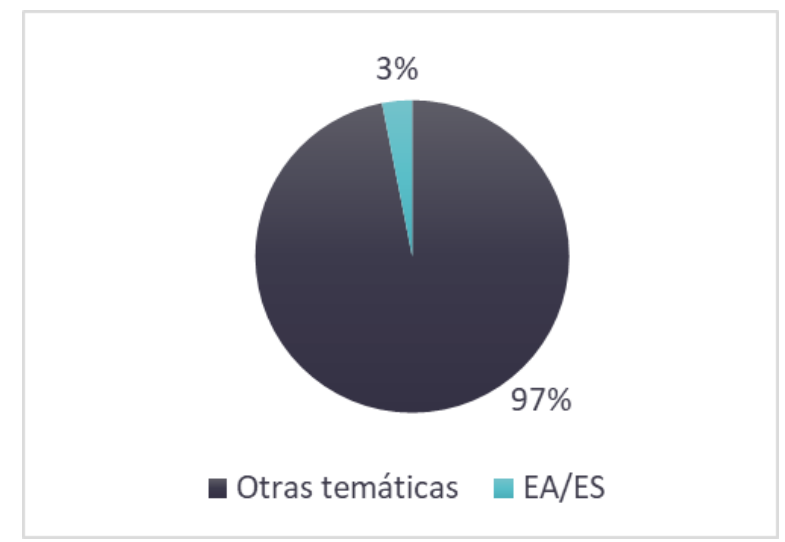

Figura 1. Presencia de las publicaciones de EA/ES entre 2014-2018

Dentro de la escasa representatividad que suponen estas temáticas en el cómputo total de artículos analizados, la presencia de estas temáticas en las diferentes revistas ha variado a lo largo del periodo de análisis, tal como se refleja en la figura 2. Exceptuando el año 2016, que supone el año donde menos publicaciones hubo sobre las temáticas en cuestión, el resto supera el 15\%, dato que, aun no suponiendo una alta representatividad, sí que muestra que estas publicaciones tienen espacios en las diferentes revistas. Destaca significativamente el año 2017 donde las publicaciones suponen un $37 \%$, lo cual se corresponde con números extraordinarios o monográficos sobre temas de EA/ES.

También podría deberse a la influencia del lanzamiento en 2015 de la Agenda 2030 (Naciones Unidas, 2015), pues hasta 2017 puede considerarse un tiempo suficiente 
para desarrollar iniciativas e investigaciones en torno a la misma, única variable diferente que podemos considerar.

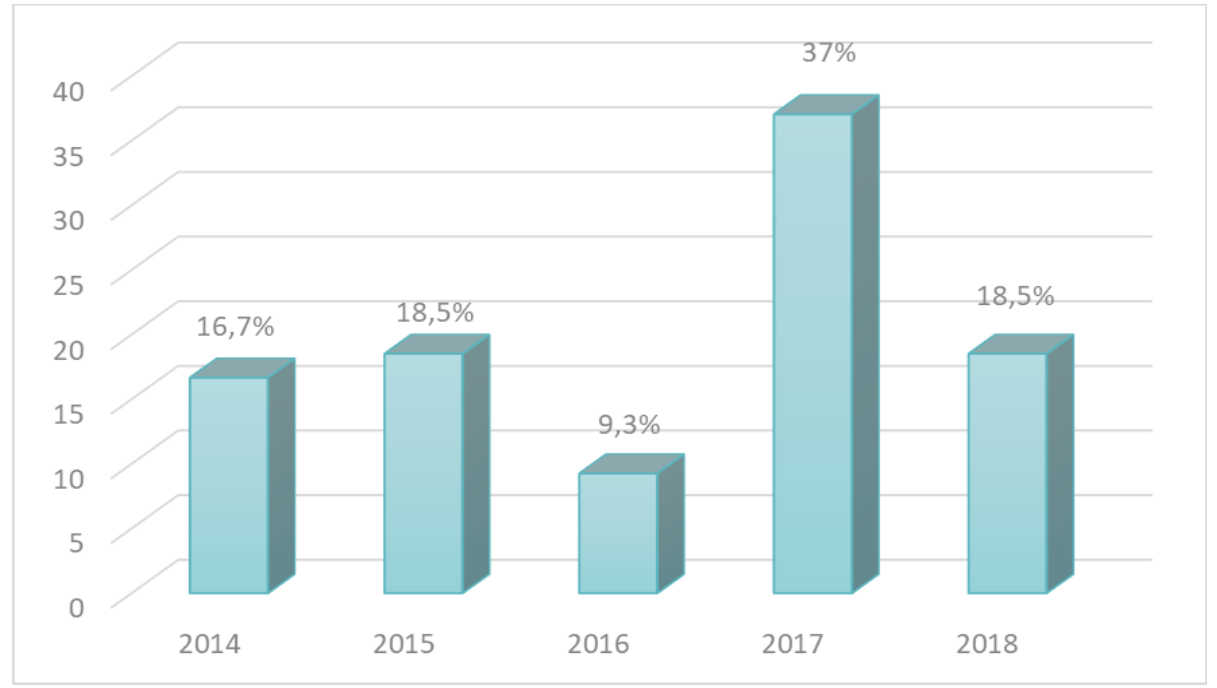

Figura 2. Presencia de EA/ES entre 2014-2018

Cuestión 2. Analizar la representatividad de las temáticas de EA/ES dentro de dichas publicaciones, así como su política y alcance.

Como ha quedado manifiesto en anteriores apartados las temáticas objeto de análisis tienen una baja representatividad en el cómputo global de artículos, no obstante, esta representatividad es diferente en cada una de las revistas de la muestra (figura 3).

Revista Iberoamericana de Educación es la publicación que mayor número de artículos ha publicado para el periodo comprendido entre 2014-2018, suponen un 33\% del total. Ello se debe, en cierta medida, a que en 2017 apareció en la misma un monográfico titulado "Desarrollo sostenible y educación superior en un mundo global" donde se presentan numerosas investigaciones e iniciativas relacionadas con EA/ES.

En segundo lugar, aparece Revista Eureka sobre Enseñanza y Divulgación de las Ciencias, donde las publicaciones de la temática de análisis suponen el 20\%, una representación muy cercana a la del caso de Enseñanza de las Ciencias donde son de 19\%. Esta cuestión puede deberse a que la Didáctica de las Ciencias, principal objeto de estas publicaciones, es un ámbito de conocimiento muy ligado a la EA/ES, considerándose asimismo uno de los principales impulsores de la misma (GarcíaGonzález, Jiménez-Fontana, Azcárate y Navarrete, 2016).

En relación a las políticas y alcance de las revistas, la tabla 1 muestra los resultados obtenidos. De las nueve revistas que forman parte de la muestra solo Revista de Didáctica de las Ciencias Experimentales y Sociales y Revista Eureka sobre Enseñanza y Divulgación de las Ciencias hacen mención explícita en su "Política y Alcance" a la EA/ES, asimismo ambas tienen una sección específica sobre estas temáticas. También la tiene Ecología Austral. A excepción de Revista Eureka, en las otras dos a pesar de tener secciones específicas destinadas a EA/ES, los artículos de estas temáticas no son significativos, como se presentaba en la figura 3. 


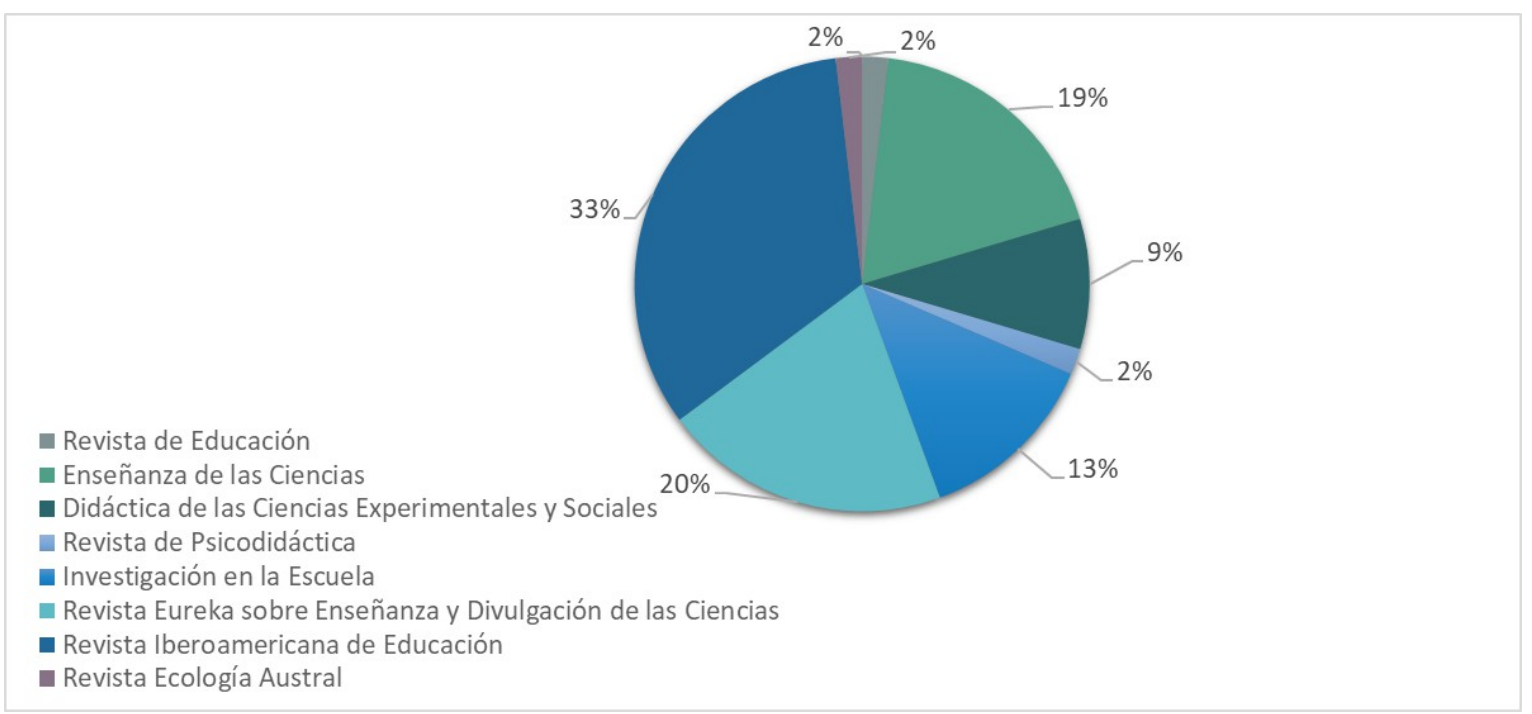

Figura 3. Representatividad de la EA/ES en las revistas analizadas.

Los resultados reflejan que estas cuestiones no forman parte de las bases y fundamentos por los que se rige la mayoría de revista analizadas. No obstante, este hecho no supone un impedimento para que acojan publicaciones de estas temáticas, aunque dada su presencia real, los artículos relacionados con la EA/ES no son fácilmente admitidos.

Tabla 1. Política, alcances y secciones de las revistas en relación a la EA/ES.

\begin{tabular}{|c|c|c|}
\hline Revista & Política y Alcances & Sección \\
\hline $\begin{array}{l}\text { Revista de } \\
\text { Psicodidáctica }\end{array}$ & $\begin{array}{l}\text { Trabajos científicos que contribuyen al avance del conocimiento } \\
\text { en el campo en el que se encuentran y se superponen la } \\
\text { psicología y la didáctica. }\end{array}$ & No \\
\hline $\begin{array}{l}\text { Bolema: Boletim de } \\
\text { Educação } \\
\text { Matemática. }\end{array}$ & $\begin{array}{l}\text { Artículos cuyo foco esté relacionado con la enseñanza y el } \\
\text { aprendizaje de la Matemática y/o el papel de la Educación } \\
\text { Matemática en la sociedad. }\end{array}$ & No \\
\hline $\begin{array}{l}\text { Enseñanza de las } \\
\text { Ciencias }\end{array}$ & $\begin{array}{l}\text { Artículos que traten de temas relevantes para la educación } \\
\text { científica y matemática y estudios que correspondan a las } \\
\text { necesidades del profesorado de ciencias y matemáticas. }\end{array}$ & No \\
\hline Revista de Educación & $\begin{array}{l}\text { Artículos sobre el conocimiento científico relacionado con el } \\
\text { ámbito de la investigación educativa, dar a conocer } \\
\text { metodologías y técnicas que se utilizan y propiciar el debate } \\
\text { sobre problemas del ámbito educativo. }\end{array}$ & No \\
\hline $\begin{array}{l}\text { Revista de Didáctica } \\
\text { de las Ciencias } \\
\text { Experimentales y } \\
\text { Sociales }\end{array}$ & $\begin{array}{l}\text { Artículos dedicados a la investigación e innovación en didáctica } \\
\text { de la Física, de la Química, de la Biología y de las Ciencias de la } \\
\text { Tierra, en Educación Ambiental y Sostenibilidad, en Promoción } \\
\text { y Educación para la Salud y en didáctica de la Historia y de la } \\
\text { Geografía. }\end{array}$ & Sí \\
\hline $\begin{array}{l}\text { Revista de } \\
\text { Investigación en la } \\
\text { Escuela }\end{array}$ & $\begin{array}{l}\text { Artículos dirigidos prioritariamente al profesorado e } \\
\text { investigadores interesados en su desarrollo y en el estudio y } \\
\text { mejora de los procesos de enseñanza de materias escolares de } \\
\text { cualquier nivel educativo, desde una perspectiva abierta a la } \\
\text { innovación, fundamentada y crítica. }\end{array}$ & No \\
\hline $\begin{array}{l}\text { Revista Eureka sobre } \\
\text { Enseñanza y } \\
\text { Divulgación de las } \\
\text { Ciencias }\end{array}$ & $\begin{array}{l}\text { Intenta contribuir al desarrollo del conocimiento en el ámbito } \\
\text { de la didáctica de las ciencias experimentales, tanto en un plano } \\
\text { teórico como aplicado. Considera temas multidisciplinares o } \\
\text { transversales, tales como la educación para la sostenibilidad, la } \\
\text { educación para la salud, la formación del profesorado etc. }\end{array}$ & Sí \\
\hline
\end{tabular}


Tabla 1. Continuación.

\begin{tabular}{|c|c|c|}
\hline Revista & Política y Alcances & Sección \\
\hline $\begin{array}{l}\text { Revista } \\
\text { Iberoamericana de } \\
\text { Educación }\end{array}$ & $\begin{array}{l}\text { Artículos que recogen las opiniones actuales más destacadas } \\
\text { sobre temas educativos y experiencias innovadoras. Y aquellos } \\
\text { que hagan avanzar el conocimiento en un área determinada } \\
\text { relacionada de la educación o investigaciones que ofrezcan } \\
\text { resultados significativos. }\end{array}$ & No \\
\hline Ecología Austral & $\begin{array}{l}\text { Publica artículos científicos en cualquier rama de las ciencias } \\
\text { ambientales. Pueden ser resultados de una investigación que } \\
\text { signifiquen un aporte original al conocimiento en ecología, o en } \\
\text { el desarrollo de una metodología y colaboraciones sobre temas } \\
\text { relacionados con los curricula de Ecología. }\end{array}$ & Sí \\
\hline
\end{tabular}

Cuestión 3. Caracterizar el tipo de trabajos publicados en el ámbito de la EA/ES.

En relación a la tipología de los artículos, en la figura 4 se aprecia con claridad que las publicaciones analizadas están orientadas principalmente a la investigación en el campo de la EA/ES, estas representan el 74\%, por lo que los investigadores y autores de estos trabajos prefieren comunicar los resultados en este sentido. Las otras dos tipologías son mucho menos significativas, de hecho, ni sumándolas llegan a la mitad de lo que suponen las investigaciones.

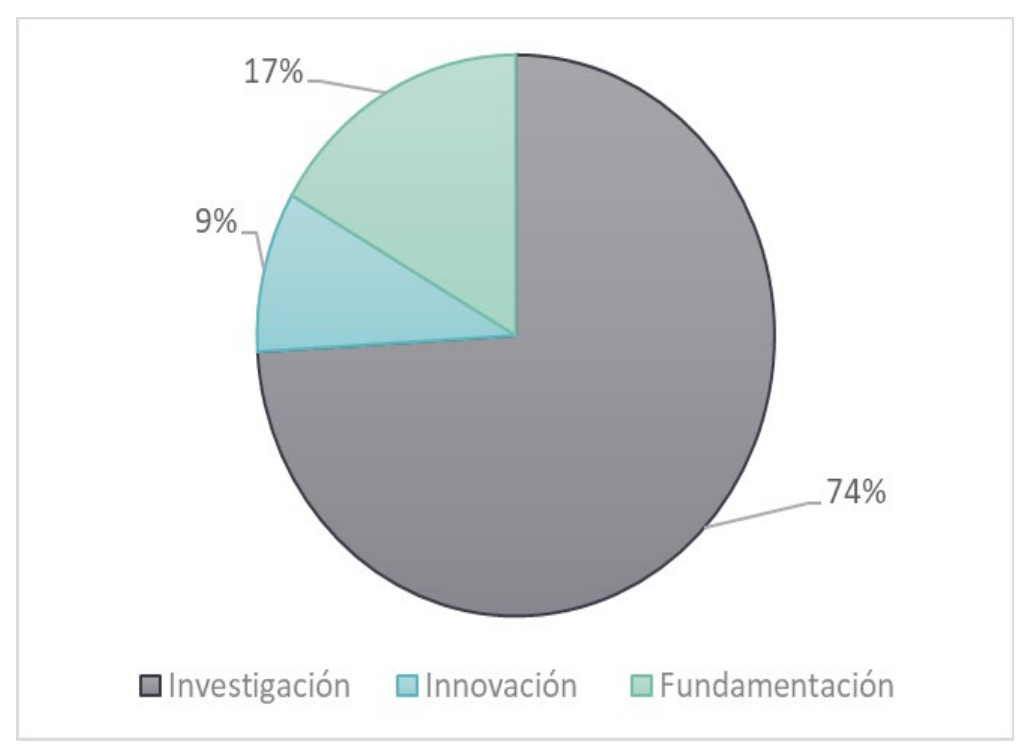

Figura 4. Tipología de las publicaciones

El dato para los artículos de fundamentación, 17\%, indica que son pocos los que ponen el centro de interés en la construcción y discusión del cuerpo teórico de la EA/ES. Por último, los relacionados con intervenciones de carácter innovador solo representan el 9\% del total.

La frecuencia de las diferentes tipologías ha variado a lo largo de los años analizados, como se aprecia en la figura 5. En este desglose puede verse como las de corte investigativo son las más representativas en el año 2017, no así en el resto de años. De hecho, en 2014 son más representativas las de innovación y en 2018 las de fundamentación. También es significativo que en 2016 solo se publicaron artículos de fundamentación, precedidos de un año 2015 donde no hubo ninguno de esta orientación. 


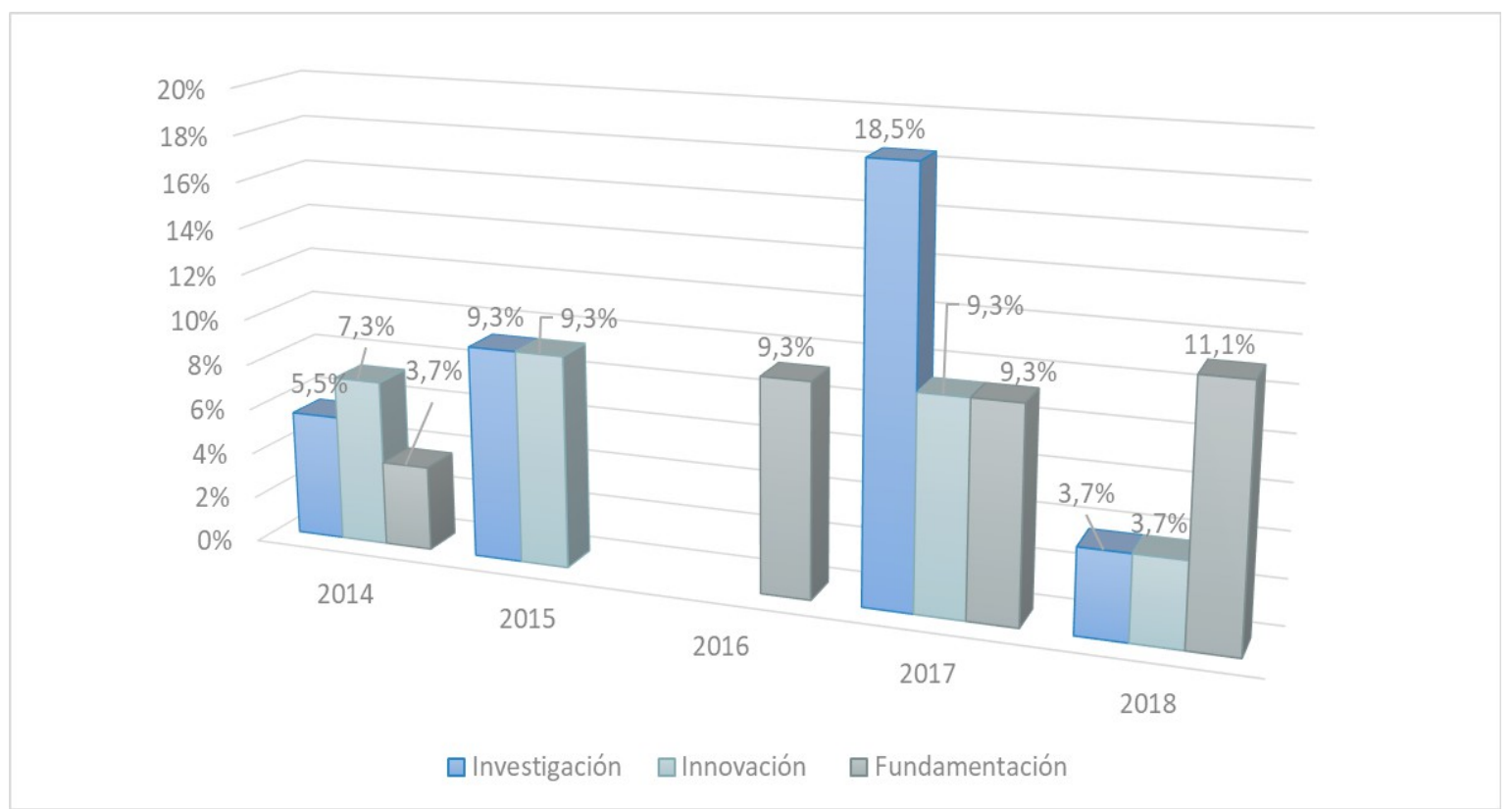

Figura 5. Orientación de las publicaciones por año analizado

\section{Conclusiones}

A modo de síntesis, los resultados obtenidos en el análisis presentado a lo largo de este estudio muestran que:

- La presencia de temáticas relacionadas con la EA/ES en el ámbito educativo y el de las ciencias experimentales durante el periodo que comprende 20142018 es muy reducido. No obstante, en todos los años de estudio hubo publicaciones de estas temáticas, lo cual indica que, en cierta medida, tienen cabida como cualquier tema de otra índole.

- Las revistas que han prestado mayor atención a estas temáticas fueron principalmente Revista Iberoamericana de Educación, seguidas por Revista Eureka sobre Enseñanza y Divulgación de las Ciencias y Revista de Educación. Asimismo, solo Revista Eureka sobre Enseñanza y Divulgación de las Ciencias, Revista de Didáctica de las Ciencias Experimentales y Sociales, y Revista de Ecología Austral, contemplan estas temáticas como parte de su política y alcance o sus secciones.

- En relación a la tipología de los artículos, son significativamente más numerosos los de investigación.

Atendiendo a las cuestiones escritas líneas arriba es necesario un incremento de publicaciones en el ámbito de la EA/ES pues es sin duda una vía para hacer llegar tanto a la comunidad científica como a la sociedad, información sobre la situación de crisis socioambiental pero también sobre posibles soluciones a la misma que se manifiestan en estos trabajos. Para ello, resulta acuciante que las revistas del ámbito educativo y del campo de las ciencias experimentales, presentes o no presentes en este estudio, sean receptivas a este tipo de trabajos. También es importante ampliar los espacios y posibilidades de publicación de estos temas. Asimismo, urge que los autores e investigadores de este campo aúnen esfuerzos por consolidar un cuerpo teórico en esta disciplina y para ello deben aumentar los trabajos de fundamentación. 
En otro orden de cosas, como hemos analizado en el marco teórico, son múltiples los problemas que tiene esta temática, aspecto que se ve auspiciado por la falta de espacios destinados a publicaciones de estas características.

Si pretendemos que la sostenibilidad en el ámbito educativo como una opción de construcción de respuestas, de soluciones orientadas (Wiek, Withycombe y Redman, 2011) no solo sea una emergencia, sino una realidad, en respuesta a la creciente preocupación por la situación de emergencia planetaria (Vilches y Gil, 2009) es necesario afrontar el reto de la actividad investigadora y su concreción en publicaciones, para hacerla crecer (Oliva, 2010). Esto es así pues las publicaciones son un instrumento con alto potencial para la comunicación entre investigadores, referente entre profesionales y la sociedad en general, considerándose interconectores elementales en el proceso de transferencia y difusión de la ciencia.

Se hace necesaria una mayor difusión científica de la rica experiencia existente en este campo educativo y se deberían alentar la EA/ES en las líneas de investigación vigentes en nuestro país (Novo y Bautista-Cerro, 2012). Es en este sentido por lo que consideramos necesaria, conveniente y oportuna la creación de una revista como Revista de Educación Ambiental y Sostenibilidad (REAyS), que puede servir de soporte y promoción de la producción científica y de las experiencias realizadas en este ámbito de conocimiento y así poder contribuir a la toma de conciencia a nivel global de la emergencia planetaria en que nos encontramos y de la necesaria interacción entre los diferentes ámbitos del conocimiento.

\section{Referencias bibliográficas}

Benayas, J., Gutiérrez, J. y Hernández, N. (2003). La investigación en educación ambiental en España. Madrid: Ministerio de Medio Ambiente.

Colom A. J. y Sureda J. (1989). La lectura pedagógica de la Educación Ambiental. Educación Ambiental. Sujeto, entorno, sistema. Salamanca: Amarú.

Echeverría, J. (2008). Transferencia de conocimiento entre comunidades científicas. Arbor, 184(731), 539-548.

Ferreras-Fernández, T. y Merlo-Vega, J. A. (2015). Repositorios de acceso abierto: un nuevo modelo de comunicación científica. La Revista de la Sociedad ORL CLCR en el repositorio Gredos. Revista de la Sociedad Otorrinolaringológica de Castilla y León, Cantabria y La Rioja, 6(12), 94-113.

Flores, R. C. (2012). Investigación en Educación Ambiental. Revista Mexicana de Investigación educativa. 55(17), 1019-1033.

García, J. E. (2002). Los problemas de la Educación Ambiental: ¿Es posible una Educación Ambiental integradora? Investigación en la Escuela, 46, 5-25.

García J. E. (2004). Educación Ambiental, constructivismo y complejidad. Sevilla: Diada Editora.

García-González, E., Jiménez-Fontana, R., Azcárate, P. y Navarrete, A. (2016). La Educación para la sostenibilidad en el ámbito universitario. Un estudio de caso múltiple en la Universidad de Cádiz. En Actas 27 Encuentros de Didáctica de las Ciencias Experimentales (pp. 227-234). 
Jiménez-Fontana, R. y García-González, E. (2017). Visibilidad de la Educación Ambiental y la Educación para la Sostenibilidad en las publicaciones españolas sobre educación científica. Revista Eureka Sobre Enseñanza y Divulgación de las Ciencias, 14(1), 271-285.

Krippendorff, K. (2013). Content analysis: an introduction to its methodology. Los Angeles / London: Sage.

López Noguer, F. (2002). El análisis de contenido como método de investigación. Revista de Educación, 4, 167-179.

Mayer, M. (1998). Educación ambiental: De la acción a la investigación. Revista Enseñanza de las Ciencias, 16(2), 217-231.

Naciones Unidas (2015). Transformar nuestro mundo: la Agenda 2030 para el Desarrollo Sostenible.

Novo, M. y Bautista-Cerro, M. J. (2012). Análisis de la incidencia de la educación ambiental para el desarrollo sostenible en las revistas científicas españolas. Revista de Educación, 358, 583-597.

Newby, P. (2010). Research Methods for Educatión. Harlow, United Kingdom: Pearson.

Oliva J. M. (2010) La educación secundaria como foco de atención en las publicaciones de didáctica de las ciencias de revistas de nuestro entorno. XXIV Encuentro de Didáctica de las Ciencias Experimentales (pp. 53-58). Baeza (Jaén).

Ortega Mohedano, F. y Galhardi, C. (2013). Propuesta metodológica para el análisis de contenido de la parrilla de televisión en Brasil: análisis de un caso práctico en el estado de São Paulo. Actas del $2^{\circ}$ Congreso Nacional sobre Metodología de la Investigación en Comunicación (1, pp. 221-239). Segovia: Universidad de Valladolid, Facultad de Ciencias Sociales, Juridicas y de la Comunicación.

Sauvé L. (1999). La educación ambiental: entre la modernidad y la posmodernidad: en busca de un marco de referencia educativo integrador. Tópicos en Educación Ambiental, 2(1), 7-25.

Vilches, A. y Gil, D. (2009). Una situación de emergencia planetaria, a la que debemos y «podemos» hacer frente. Revista de Educación, № Extra 2009, 101-122.

Wiek, A., Withycombe, L. and Redman, C. L. (2011). Key competencies in sustainability: a reference framework for academic program development. Sustainability Science, 6(2), 203-218. DOI: https://doi.org/10.1007/s11625011-0132-6. 